\title{
Asymmetrischer Wiederaufbau in Städten nach Katastrophen. Das Lower Ninth Ward in New Orleans nach Orkan Katrina
}

\author{
Mark Kammerbauer
}

Eingegangen: 28. August 2013 / Angenommen: 19. August 2014 / Online publiziert: 17. September 2014

(C) Springer-Verlag Berlin Heidelberg 2014

\begin{abstract}
Zusammenfassung Wenn durch Hochwasserereignisse ausgelöste Katastrophen Schäden in Städten hervorrufen, stellt der Wiederaufbau danach ein komplexes Handlungsfeld dar, das den städtischen Raum, intervenierende staatliche Institutionen und nicht zuletzt betroffene Bürger umfasst. Es ist möglich, dass der Wiederaufbau nicht gleichmäßig, sondern asymmetrisch verläuft. Im Fall der US-amerikanischen Stadt New Orleans, 2005 vom Orkan Katrina schwer getroffen, lässt sich dies seitdem beobachten, insbesondere im Quartier Lower Ninth Ward. Hier sollte eigentlich ein auf Hauseigentümer ausgerichtetes Wiederaufbauprogramm den Bürgern die Rückkehr erleichtern. Warum konnten die Pläne und Programme des Wiederaufbaus das Entstehen einer Asymmetrie beim Wiederaufbau des Lower Ninth Ward nicht verhindern? Der Beitrag stellt fest, dass diese Pläne und Programme nicht adäquat auf städtebauliche und soziale Rahmenbedingungen reagierten und nicht auf die Verwundbarkeit der betroffenen Stadtbewohner ausgerichtet waren. Der Beitrag enthält hierzu mittels quantitativer und qualitativer Methoden 2007 und 2009 erhobene empirische Daten. Das Ziel ist, wissensbasierte Planungsempfehlungen zu unterstützen, die auf die Verwundbarkeit betroffener Bürger eingehen, und zu einer Diskussion über gerechten und nachhaltigen Wiederaufbau in und von Städten nach Katastrophen anzuregen.
\end{abstract}

Dr.-Ing. M. Kammerbauer, MSc, Dipl.-Ing. ( $\bowtie)$

Technische Hochschule Nürnberg,

Keßlerplatz 12,

90489 Nürnberg, Deutschland

E-Mail: info@transarchitecture.org

Dr.-Ing. M. Kammerbauer, MSc, Dipl.-Ing.

University of Queensland,

St Lucia QLD 4072,

Brisbane, Australia
Schlüsselwörter Wiederaufbau · Hochwasser · Katastrophe · Verwundbarkeit · Stadtplanung

\section{Asymmetrical Recovery in Cities After Disaster. The Lower Ninth Ward in New Orleans After Hurricane Katrina}

\begin{abstract}
When flood events trigger disaster in cities, the recovery process constitutes a complex field of activity covering urban space, state institutions, and impacted citizens. Recovery may not occur evenly, but instead, asymmetrically. In the case of the city of New Orleans in the USA, heavily hit by Hurricane Katrina in 2005, this can be observed particularly in the city's Lower Ninth Ward. Here, a rebuilding program oriented towards homeowners was supposed to enable citizens to return. Why couldn't the plans and programs for recovery prevent the emergence of asymmetric recovery in the Lower Ninth Ward? This paper shows that these plans and programs didn't adequately respond to urban spatial and social conditions and weren't oriented towards the vulnerability of impacted citizens. For this purpose, empirical data collected via quantitative and qualitative methods in 2007 and 2009 are featured here. The aim is to support knowledge-based planning recommendations that address the vulnerability of impacted citizens and to contribute to a discussion on just and sustainable recovery in and of cities after disaster.
\end{abstract}

Keywords Disaster recovery - Floods - Disaster · Vulnerability $\cdot$ Urban planning 


\section{Einleitung}

Dieser Beitrag behandelt den Wiederaufbau in Städten nach Naturkatastrophen am Beispiel der US-amerikanischen Stadt New Orleans nach dem verheerenden Orkan Katrina im Jahr 2005. Der Fokus wird dabei auf das Quartier Lower Ninth Ward gerichtet, das auch als Fallstudie dient. Dieser Teil der Stadt erlangte nach der Katastrophe traurige Berühmtheit und kann stellvertretend für die Probleme der Stadt beim Wiederaufbau betrachtet werden. Aufgrund der Katastrophe, die durch das extreme klimatische Ereignis und dem damit verbundenen Hochwasser ausgelöst wurde, musste die gesamte Bevölkerung aus der Stadt evakuiert werden und ist seitdem noch nicht in Gänze zurückgekehrt. Das Lower Ninth Ward ist ein Stadtteil, der von dieser unvollständigen Rückkehr erheblich betroffen ist. Auch machten durch das Hochwasser entstandene Schäden an Wohnbauten weitreichende Wiederaufbaumaßnahmen notwendig.

Der Fortschritt beim Wiederaufbau ist jedoch trotz der damit verbundenen bundesstaatlichen Finanzierung und staatlichen und lokalen Pläne und Programme begrenzt. Der sehr beschränkt erfolgreiche Wiederaufbau im Lower Ninth Ward ergibt außerdem ein asymmetrisches Bild - im Sinne von ungleichmäßig und als Gegensatz zu symmetrisch. Es dauerte Jahre, bis die fast allgegenwärtigen Ruinen und Schutthalden weggeräumt waren. Dem Beobachter bietet sich noch immer eine differenzierte Situation. So sieht man im südlichen, dem Mississippi näher gelegenen Teil historische Holzhäuser im Wechsel mit leer geräumten Grundstücken. Von hier bis zum nördlichen Teil wird nicht nur das Straßenniveau tiefer. Hier befanden sich vor Katrina mehrheitlich Häuser, die nach dem Zweiten Weltkrieg gebaut wurden. Nun überwiegen die leer stehenden Grundstücke zum Teil so stark, dass man nicht mehr den Eindruck hat, in einer Stadt zu stehen. Ein Straßenzug mit Neubauten stellt die Ausnahme in dieser de-urbanisierten Landschaft dar.

Die physische Wiederherstellung von Bauten und Infrastruktur kann jedoch nur unzureichend darüber Auskunft geben, was die Gründe für die Probleme des Wiederaufbaus sind. Warum konnten die Pläne und Programme nicht die entstandene Asymmetrie im Wiederaufbau des Lower Ninth Ward verhindern? Was sind die tief liegenden Gründe für diese Situation? Wie reagieren gemeinnützige und Non-Profit-Organisationen darauf? Die Hypothese dieses Beitrags besteht darin, dass Pläne und Programme des Wiederaufbaus nur bedingt auf diejenigen städtebaulichen und sozialen Rahmenbedingungen und spezifischen Charakteristiken eingingen, die sich in der Katastrophe als verwundbarkeit herausstellten.

Die finanzielle Hilfe der amerikanischen Bundesregierung zum Wiederaufbau basiert auf einem bestehenden gesetzlichen Rahmen, dem „Stafford Act“, der auf eine
Rückkehr zu den Bedingungen des Status quo ante abzielt. Der betroffene Staat Louisiana entwickelte in diesem Zusammenhang ein Wiederaufbauprogramm, das „Road Home Program", das im Wesentlichen betroffenen Hausbesitzern mit unversicherten Schäden Fördergelder zum Wiederaufbau anbot. Jedoch musste es nach dem Anlaufen um eine Rückkaufoption, den sogenannten „Buyout“, ergänzt werden. Die Stadt New Orleans bedient sich einer städtischen Behörde, der „New Orleans Redevelopment Agency“ (NORA), um auf die staatliche Initiative planerisch reagieren zu können. $\mathrm{Zu}$ den Aufgaben von NORA zählten der Kauf und Verkauf von leer stehenden und verlassenen Grundstücken. Diese Kompetenz wird beim Wiederaufbau innerhalb des dafür geschaffenen „Lot Next Door Program“ genutzt, um durch den Buyout vom Staat Louisiana aufgekaufte Grundstücke strategisch für den Wiederaufbau zu nutzen. Dies sollte durch einen präferierten Verkauf dieser Grundstücke durch NORA an die jeweiligen benachbarten Hauseigentümer geschehen. Im Lower Ninth Ward scheinen diese Maßnahmen und Institutionen nur sehr bedingt erfolgreich zu sein. Gleichzeitig agieren hier Non-Profit-Organisationen, die betroffenen Bürgern bei ihren Wiederaufbaubemühungen Unterstützung anbieten.

Im Folgenden werden der Stand der Forschung und die theoretische Grundlage zur Untersuchung des Wiederaufbaus von Städten nach Katastrophen dargestellt (Kapitel 2). Danach werden der Planungskontext und die wesentlichen Planungen und Programme beim Wiederaufbau von New Orleans und dem Lower Ninth Ward nach Katrina beschrieben (Kapitel 3), darunter Stafford Act, Road Home Program und Lot Next Door Program. In Kapitel 4 wird die Methodologie für die empirische Fallstudie im Lower Ninth Ward entwickelt und diskutiert. Eine Analyse der im Lower Ninth Ward in 2007 und 2009 vom Autor erhobenen empirischen Daten soll Einblick in die Ursachen des asymmetrischen Wiederaufbaus in Bezug auf Verwundbarkeit der Betroffenen und die jeweiligen Planungen und Programme geben (Kapitel 5). Eine Zusammenfassung versucht, aus den gewonnenen Erkenntnissen Lehren für zukünftige Planungen für den Wiederaufbau in und von Städten nach Katastrophen zu ziehen und Potenziale und Grenzen der Übertragbarkeit aufzuzeigen (Kapitel 6).

\section{Stand der Forschung zum Thema Wiederaufbau nach Katastrophen}

\subsection{Katastrophenforschung und Verwundbarkeit}

Ein wesentlicher Standpunkt der Katastrophenforschung besteht darin, dass eine Fokussierung auf das physikalische Risiko klimatischer Ereignisse nicht den tatsächlichen Ursachen des Risikos gerecht wird. Die tatsächlichen Ursa- 
chen sind vielmehr in sozioökonomischen und politischen Prozessen begründet, die dazu führen, dass Menschen sich in verwundbaren Situationen befinden. Dieser Umstand erschwert Betroffenen, die Einwirkungen klimatischer Ereignisse zu bewältigen. Diejenigen, die am meisten unter klimabezogenen Katastrophen zu leiden haben, befinden sich an den Rändern der Gesellschaft. Sie entscheiden sich nicht dafür, sich einem Risiko auszusetzen - stattdessen sind sie oft genug gezwungen, an risikoreichen Orten zu wohnen, da ihnen Orte mit geringerem Risiko nicht zur Verfügung stehen oder nicht erschwinglich sind (Gaillard/ Glantz/Kelman et al. 2014: 1, 14).

Anstatt Katastrophen als physische Ereignisse zu definieren, deren Problemlösung technologisch erfolgen könne, sollten also Katastrophen als das Ergebnis einer komplexen Interaktion betrachtet werden, gekennzeichnet durch einen potenziellen physischen Schadensfall (z. B. Flut, Trockenheit, Feuer, Erdbeben und Stürme) und die Verwundbarkeit einer Gesellschaft sowie ihrer Infrastruktur, Wirtschaft und (gebauten) Umwelt. Unter dieser Voraussetzung können und sollen Naturkatastrophen als ,un-natürliche' Katastrophen verstanden werden. Dabei ist die Definition von Verwundbarkeit schwierig; die Literatur umfasst verschiedene Definitionen, Konzepte, Methoden und Systematiken. Verschiedene (Forschungs-)Disziplinen haben ihre jeweils eigenen Ansätze zur Bedeutung der Verwundbarkeit entwickelt, die zudem als multidimensional, differenziell, skalenabhängig und dynamisch betrachtet wird (Birkmann 2006: 10 f.). Hinsichtlich sozialer Verwundbarkeit mangelt es ebenso an einer gemeinsamen Definition. Das Konzept der sozialen Verwundbarkeit kann ökonomische und physische Aspekte umfassen, solange diese Ausdruck einer sozial konstruierten Verwundbarkeit sind. Die Analyse von Schadensmustern kann dabei zur Ermittlung gegenwärtiger wie potenzieller zukünftiger Verwundbarkeiten beitragen (Birkmann 2006: 13 f.).

\subsection{Doppelte Struktur der Verwundbarkeit}

Nach Chambers (1989: 1) besteht eine mögliche Konzeptualisierung der Verwundbarkeit darin, dass sie eine externe und eine interne Seite hat. Die interne Seite ist durch mangelnde Abwehrmöglichkeiten oder fehlende Bewältigungskapazität gekennzeichnet. Die externe Seite umfasst die Risiken, Schocks und Stress, denen ein Individuum oder ein Haushalt ausgesetzt sind. Aus diesem Ansatz entwickelt Bohle (2001) das Konzept der doppelten Struktur der Verwundbarkeit. Die externe Seite wird hierbei als die strukturelle Dimension der Verwundbarkeit und des Risikos bezeichnet. Die interne Seite verweist auf die Bewältigungsstrategien für schädliche Auswirkungen von Wandlungsprozessen ökonomischer und ökologischer Art.

Die jeweiligen Bewältigungsstrategien können sich dabei nach Region, Gesellschaft, Gemeinschaft, sozialer
Zugehörigkeit, Geschlecht, Alter und saisonalem sowie historischem Zeitpunkt unterscheiden. Verwundbarkeit kann sich verändern, beispielsweise wenn sich öffentliche Dienstleistungen verbessern (Chambers 1989: 3 f.). Die externe und interne Seite der Verwundbarkeit können dabei als Bestandteile einer interaktiven und dialektischen Beziehung angesehen werden (Bohle 2001: 3). Für hochgradig verwundbare Populationen kann durch eine Einbindung in soziale Netzwerke gegenseitigen Vertrauens die Resilienz gegenüber ökonomischen und ökologischen Risiken erhöht werden. Dies ist aber abhängig von der politischen Ökonomie der jeweiligen Region sowie der Art und Weise, wie verschiedene Populationen in die jeweiligen Strukturen und Dynamiken von Gesellschaft, Wirtschaft und Politik eingebunden sind (Bohle 2001: 3).

\subsection{Wiederaufbau und soziales Kapital}

Pohl (2010) zeigt, wie der Wiederaufbau in den Katastrophenzyklus eingeordnet werden kann und welche Phasen sich unterscheiden lassen. Der Katastrophenzyklus umfasst dabei Vorbereitung, Reaktion, Erholung und Vorsorge. Auslöser ist ein „zentrales Ereignis“ (Pohl 2010: 14). Die jeweiligen Übergänge sind nicht eindeutig. Für den eigentlichen Wiederaufbau können vier Phasen definiert werden: unmittelbare Nothilfe, Wiederherstellung provisorischer Lebensverhältnisse, Wiederaufbau I und Wiederaufbau II (vgl. Pohl 2010: 14 f.). Die Dauer der unmittelbaren Nothilfe hängt dabei von der Schwere der Katastrophe ab. $\mathrm{Zu}$ den provisorischen Lebensverhältnissen zählt auch die Situation von Evakuierten. Bereits in der Phase Wiederaufbau I sollen Möglichkeiten und Gelegenheiten für ,strukturelle Veränderungen“ (Pohl 2010: 15) genutzt werden, die in der Phase Wiederaufbau II den Weg für eine „langfristig positive Entwicklung" (Pohl 2010: 15) bereiten können. Es wird gefordert, dass der Wiederaufbau über eine reine Rekonstruktion hinausgehen solle: „Dabei ist die Reduktion des Risikos künftiger Katastrophen als integraler Bestandteil der Wiederaufbauplanung zu bezeichnen. Das Leitbild ,building back better' impliziert, dass nicht der Status quo ante das Kernziel sein sollte, sondern eine Verringerung der Vulnerabilität im Kontext von Katastrophen anzustreben ist" (Pohl 2010: 13).

Der Wiederaufbau ist abhängig von der Rückkehr der Bevölkerung und der Wiedererrichtung städtischer Infrastruktur und Dienstleistungen. Die Dauer des Wiederaufbaus wird bedingt durch Bevölkerungsverlust und Evakuierung, sie richtet sich nach dem Grad der Zerstörung, Verfügbarkeit von Ressourcen sowie vorbereitender Planung. Ausdrücklich betont wird die Rolle staatlicher Institutionen, die an der Umsetzung des Wiederaufbaus beteiligt sind (Vale/ Campanella 2005: 13). 
$\mathrm{Zu}$ den Faktoren, die die Folgen von Extremereignissen beeinflussen können, zählen auch schlechtes oder unzureichendes Management beim Wiederaufbau, dessen Ursachen mit den sozialen, kulturellen und ökonomischen Aspekten des Extremereignisses in Verbindung stehen (IRDR 2011: 12). In diesem Zusammenhang spielt soziales Kapital eine wichtige Rolle. Putnam (2007: 124 f.) betrachtet soziales Kapital als Konzept, das auf Koordination und Kooperation zu beiderseitigem Nutzen abzielt. Beispielhaft dafür sind Non-Profit-Organisationen. Bohle (2001) verweist ebenso auf die Bedeutung von sozialen Netzwerken gegenseitigen Vertrauens im Rahmen der Verwundbarkeit und seiner doppelten Struktur. Dynes (2006) interpretiert dabei die Akquirierung der Mithilfe von Freiwilligen im Kontext einer Katastrophe als das Ergebnis mangelnder Effektivität der verantwortlichen Institutionen. Dieser Umstand wird als Grund dafür betrachtet, Unterstützung von außerhalb einer Gemeinschaft als sozialer Einheit zu erhöhen (Dynes 2006: 13). Freiwilligenhilfe wird als Beispiel für eine organisierte Reaktion auf Katastrophen betrachtet, die gewisse Aktivität fördert, andere wiederum einschränken kann.

\subsection{Der Stadtbegriff in sozialräumlicher Perspektive}

Da die gesellschaftliche und politische Struktur der Verwundbarkeit wichtig ist, spielt eine Rolle, was von wem für wen wieder aufgebaut wird. Zur wissenschaftlichen Eingrenzung des Stadtbegriffs sind städtebauliche Leitbilder dienlich, die eine Einordnung der ,amerikanischen Stadt“ als eigenständigen Beobachtungsgegenstand zulassen. Diese entstammen sozialräumlichen Forschungsperspektiven, die sich mit der dialektischen Interaktion zwischen gebautem Raum und seinen Bewohnern befassen (vgl. Gottdiener/ Hutchison 2006). Ebenso denkbar sind vergleichbare kulturelle und städtebauliche Leitbilder wie ,europäische Stadt“ oder „chinesische Stadt“ (vgl. Hassenpflug 2009). Mit den Begriffen Urbanität und Ruralität können „,bestimmte, klar definierbare soziokulturelle Leistungen des städtischen und ländlichen Raums“ (Hassenpflug 2006: 57) bezeichnet werden. Allerdings ist der Stadt-Land-Dualismus der europäischen Geschichte nicht ohne Weiteres auf Nordamerika zu übertragen. Hier wird ein Dualismus von ,Zivilisation und Wildnis“ erkannt, der sich von den historischen, kulturellen Gegensätzen „,ständischer und moderner Gesellschaft“ in Europa (Hassenpflug 2006: 48) unterscheidet. Es kann also eine Einordnung nicht nur hinsichtlich der Stadt, sondern auch der Verbindung von Stadt und (Um-)Land vorgenommen werden. Kennzeichen der ,amerikanischen Stadt“ ist das Primat der ökonomischen Zentralität, die bei der Immobilienentwicklung einen wichtigen Indikator für die Interventionen staatlicher Institutionen im städtischen Raum darstellt. Historisch betrachtet entstand eine funktionale und sozioökonomische Differenzierung räumlicher Mus- ter; beispielhaft sind hierfür die Entkopplung von Arbeit und Arbeitsplatz, Zonierung sowie Suburbanisierung (Hassenpflug 2006). Da Hauseigentum auf den Zugang zu Ressourcen hinweist, kann die ,räumliche Verteilung von arm und reich“ (Gottdiener/Hutchison 2006: 39) dazu dienen, sozioökonomische Muster im Stadtraum nachzuzeichnen. So sind landesweit in den USA $65,5 \%$ der Wohneinheiten von ihren Eigentümern bewohnt. In den größten Städten des Landes ergibt sich jedoch ein anderes Bild: In New York City beträgt dieser Anteil 32,3\%, in Los Angeles 38\%, in Chicago 46,1\%, in Houston 45,9\% und in New Orleans betrug er vor Orkan Katrina 47\% (US Census 2012; Louisiana Recovery Authority 2006: 17). Hinzu kommt das Phänomen der räumlichen Segregation, die mit historischen Praktiken der ethnischen Segregation sowie stadträumlichen Beeinträchtigungen wie Umweltverschmutzung in Verbindung steht. Städte sind jedoch nicht ausschließlich Orte des Risikos. Sie können ebenso Schutz bieten: durch lokales Wissen im Umgang mit Gefahren, vorbereitende Planung, Vermeidung der Abhängigkeit von Einzelressourcen, soziale Dienstleistungen, Versicherungen sowie Bürgerbeteiligung (Pelling 2003: 14).

Die Katastrophenforschung ermöglicht über das Konzept der Doppelstruktur der Verwundbarkeit nach Bohle (2001) eine wissenschaftliche Einordnung des Risikos und der Katastrophen-Bewältigungskapazität der Betroffenen. Der Wiederaufbau lässt sich als Bestandteil des Katastrophenzyklus einordnen. Er sollte nach Pohl (2010) möglichst zur Verminderung der Verwundbarkeit beitragen. Auch das ,Management' des Wiederaufbaus und die Rolle des sozialen Kapitals stehen in einem Zusammenhang. So kann nicht nur auf die Planungen und Programme staatlicher Institutionen zum Wiederaufbau verwiesen werden, sondern auch auf die Art und Weise, wie soziales Kapital zum Wiederaufbau beiträgt.

Städtebauliche Leitbilder erlauben eine Einordnung und Beschreibung des damit verbundenen stadträumlichen Kontextes. In Verbindung gebracht kann man erkennen, warum urbane Populationen verwundbar sind, wo sie leben und welche institutionellen Instrumente bestehen, um den Wiederaufbau nach einer Katastrophe zu befördern.

Mit diesem Vorwissen ist nun eine eingehendere Betrachtung des Planungskontextes in New Orleans und Lower Ninth Ward möglich.

\section{Planungskontext Katrina - USA, New Orleans, Lower Ninth Ward}

Die Planungen und Programme für den Katastrophenfall in den USA und New Orleans stehen im Zusammenhang mit dem staatlichen Katastrophenschutz (Perry/Quarantelli 2005; Rodriguez/Quarantelli/Dynes 2007). Katastrophen 
werden als Störung sozialer Ordnung und des Alltagslebens aufgefasst. Die Rolle des Staates besteht in der Aufrechterhaltung und Wiedererrichtung dieser Ordnung. Die institutionelle Intervention orientiert sich am auslösenden Moment, dem Disaster Trigger, wobei nach sozialen, politischen, ökonomischen oder natürlichen und/oder technologischen Ursachen differenziert wird (Dombrowsky 1998: 20). Innerhalb der dezentralisierten institutionellen Struktur der USA hat sich ein umfassendes System des „Comprehensive Emergency Management" entwickelt (Stallings 1998; Perry/Quarantelli 2005; Stallings 2005). Es besteht dabei eine Einteilung in Phasen, die für den Katastrophenschutz grundlegend ist: Planung, Vorbereitung, Reaktion sowie Erholung/Wiederaufbau. Die Planung sollte im Idealfall vor einer Katastrophe stattfinden und notwendige Aufgaben, verfügbares Personal und Ressourcen umfassen. Die zentrale Gesetzgebung für das Katastrophenmanagement in den USA ist der „Robert T. Stafford Disaster Relief and Emergency Assistance Act“" (kurz: Stafford Act) ${ }^{1}$. Hier wird die Notwendigkeit beschrieben, betroffene Communities mithilfe staatlicher Institutionen beim Wiederaufbau und zur Wiederherstellung zerstörter Gebiete zu unterstützen, damit sie baldmöglichst wieder ein ,normales' Leben führen können. Der eigentliche Wiederaufbau ist beeinflusst von Planungsstand, Bauordnung und Baugesetzen sowie dem Zusammenspiel zwischen öffentlichen und privaten Initiativen.

Orkan Katrina sowie der kurz darauf folgende Orkan Rita verursachten allein im Staat Louisiana $75 \%$ aller Schäden an Wohnbauten in der betroffenen Region entlang der Golfküste der USA (Weiss 2007). In Louisiana befindet sich auch die Stadt New Orleans. Die Regierung des Staates gründete die „Louisiana Recovery Authority“ (LRA), um die Wiederaufbaubemühungen zu koordinieren und zu leiten. Die LRA entwickelte das Road Home Program, um Hauseigentümer dazu zu bewegen, den Wiederaufbau ihrer Häuser zu betreiben, unabhängig von Ethnie, Klasse oder Wohngegend. Mit einer Fördergeldsumme von \$ 10,4 Mrd. wurde das Programm 2006 eingeführt. Hauseigentümer, die ihr Grundstück bewohnen, standen dabei im Fokus der Bemühungen. Es bestand die Absicht, kleinere Mietgrundstücke (Small Rental Property) und bezahlbare Wohnungen ebenfalls zu unterstützen. Das Road Home Program bot finanzielle Förderung basierend auf dem Wert des Grundstücks vor dem Orkan sowie der Höhe unversicherter Schäden oder anderweitiger Formen der Wiederaufbauförderung (Czerwinski 2007: 5 ff.). Im Laufe der Programmlaufzeit wurde es mehrfach modifiziert. So wurden insgesamt vier verschiedene Optionen für Hauseigentümer angeboten: Reparatur, Wiederaufbau, Umzug oder Verkauf. Dabei bedeutet die Option Umzug, dass man ein Grundstück verkauft, jedoch

\footnotetext{
${ }^{1} \mathrm{http} / /$ www.fema.gov/pdf/about/stafford_act.pdf (03.06.2014).
}

im Staat Louisiana verbleibt. Die Option Verkauf ${ }^{2}$ beinhaltet, dass der Staat Louisiana das Grundstück von den Betroffenen aufkauft, und zwar zu $60 \%$ des Wertes vor dem Orkan abzüglich weiterer möglicher Förderung bzw. den Kosten unversicherter Schäden. ${ }^{3} \mathrm{Zu}$ fördernde Objekte mussten zum Zeitpunkt Katrinas als Hauptwohnung genutzt worden sein und ein freistehendes Gebäude mit einer oder maximal zwei Wohneinheiten enthalten. Sie mussten zudem von der bundesstaatlichen Katastrophenschutzbehörde Federal Emergency Management Agency (FEMA) entweder als zerstört oder schwer beschädigt eingestuft worden sein. Die maximale Fördersumme betrug $\$ 150.000$.

45.000 Anträge auf Road-Home-Förderung führten zu Vertragsabschlüssen. Zirka 40.000 Hauseigentümer entschieden sich dafür, die Förderung für den Wiederaufbau in New Orleans zu nutzen, die übrigen entschieden sich dagegen (GNOCDC 2009: 13). Es wurde zudem erwartet, dass die Zahl der Buyouts zunehmen würde, wenn die Betroffenen die endgültigen Förderzusagen erhalten hätten (City of New Orleans 2010: 6.26). Die an den Staat verkauften Grundstücke in der Stadt New Orleans wurden von einer Behörde der Stadt akquiriert, der New Orleans Redevelopment Authority (NORA). Diese Behörde existierte bereits vor dem Orkan Katrina und besaß neben planerischen Befugnissen auch das Recht, Grundstücke zu erwerben und weiterzuverkaufen. Vor und nach Katrina handelte es sich im Wesentlichen um leer stehende und verlassene Grundstücke. NORA entwickelte ein Programm für den Umgang mit den Buyout-Grundstücken und um gleichzeitig planerische Ziele des Wiederaufbaus zu unterstützen: das Lot Next Door Incentive Program Management (Lot Next Door Program). Es zielt hauptsächlich auf Hauseigentümer ab, die ein benachbartes Grundstück, über das NORA verfügt, kaufen wollen. Dadurch können die Fläche und der Wert des eigenen Grundstücks vergrößert und die Rückkehrrate in einer betroffenen Wohngegend (neighborhood) (rechnerisch) nach oben korrigiert werden.

Es gab nach Katrina keinen verfügbaren Plan für den Wiederaufbau der Stadt. Nach einer chaotischen Phase verschiedener Planungsinitiativen, darunter die Bring New Orleans Back Commission (BNOB) des damaligen Bürgermeisters Ray Nagin und des New Orleans Neighborhoods Rebuilding Plan des City Council, entstand der Unified New Orleans Plan (UNOP) der Greater New Orleans Foundation als kohärenter Planvorschlag, der auch einen Wiederaufbauplan für die Stadt enthielt. Dieser Citywide Strategic Recovery and Redevelopment Plan (Citywide Plan) wurde von ansässigen Stadtplanern, Universitäten und Architek-

\footnotetext{
${ }^{2}$ Für die Optionen Umzug und Verkauf wird im Beitrag nachfolgen der Begriff Buyout verwendet.

${ }^{3}$ Vgl. http://www.lpb.org/programs/LApublicsquare//LRARoadHome. $\operatorname{pdf}(02.06 .2014)$.
} 
turbüros in einem partizipativen Planungsprozess entwickelt (City of New Orleans o. J.a: 13). Der Citywide Plan definiert Prioritäten des Wiederaufbaus für einen Zeitraum von fünf bis zehn Jahren. Die Absicht ist, den Wiederaufbau dahingehend zu unterstützen, dass Stabilität, Wachstum und Wohlstand verwirklicht werden. Jedoch wird im Citywide Plan auch festgestellt, dass nicht alles in der Stadt auf einmal wiederaufgebaut werden kann und Ressourcen nicht vorschnell dort eingesetzt werden sollten, wo Bewohner sich eventuell entschließen wegzuziehen (City of New Orleans o. J.a: 9 ff.). Aus diesem Grund wurden Politikbereiche (Policy Areas) definiert, die eine Abstufung von Prioritäten im Wiederaufbau beinhalten, wobei Policy Area A und $\mathrm{B}$ geringes oder moderates Risiko und hohe bis moderate Rückkehrraten auszeichnen und Policy Area $\mathrm{C}$ von hohem Risiko und niedrigen Rückkehrraten betroffen ist (City of New Orleans o. J.a: 65).

Um diese planerischen Rahmenbedingungen besser verstehen zu können, ist ein Blick auf die betroffene Stadt notwendig. In New Orleans siedelten sich Menschen entlang des Mississippi zunächst auf natürlich höher liegenden Bereichen an. Die Trockenlegung der angrenzenden Sümpfe im frühen 20. Jahrhundert begünstigte die Entwicklung von Quartieren innerhalb des lokalen, damals jedoch ethnisch segregierten Raums. Im Stadtraum sind historische Phasen der Stadtentwicklung ablesbar, die den Ursprung als Hafenstadt und Kolonie Frankreichs ebenso aufzeigen wie das Wachstum flussaufwärts nach dem Kauf der französischen Territorien durch die USA. Ein wesentlicher Einschnitt war die Drainage der umliegenden Sumpfgebiete durch ein stadtweites Pumpensystem. Dies ermöglichte nicht zuletzt die Immobilienentwicklung dieser trockengelegten Bereiche zwischen der historischen Stadt und dem großen, nördlich gelegenen Binnensee Lake Pontchartrain (City of New Orleans 2010: 6.9). Die eigentliche Stadt wird seit 1954 als „Orleans Parish“ identifiziert. Sie bildet den Mittelpunkt der New Orleans Metropolitan Area, die umliegende suburbane Verwaltungseinheiten umfasst. Vor Katrina betrug die Bevölkerung der Metropolregion zirka 1.300.000 Einwohner, davon 450.000 in Orleans Parish. Die Stadt ist zudem seit 1999 in 13 Planning Districts eingeteilt, die insgesamt über 70 Neighborhoods als lokal identifizierbare Bereiche umfassen. ${ }^{4}$ Der historische Begriff des Ward (Bezirk) findet sich ebenfalls noch in der Bezeichnung der unterschiedlichen Stadtquartiere.

Die Trockenlegung und Urbanisierung der umliegenden Sumpfgebiete wurde durch das Flutschutzsystem der Stadt ermöglicht, jedoch führte dies auch zu Absenkung in diesen Bereichen. Aus diesem Grund liegen Teile der Stadt unter-

\footnotetext{
${ }^{4}$ Vgl. http://www.nola.gov/getattachment/44f10e2f-f23a-4cd9-aa9a$145 \mathrm{~d} 9309 \mathrm{e} 1 \mathrm{de} /$ Vol-3-Ch-3-Previous-Planning-and-Charter-Amendment/ (12.08.2104)
}

halb des Meeresspiegels (Colten 2005: 39). Die Stadt ist seit ihrem Entstehen von Orkanen bedroht. Nach Orkan Betsy 1965 plante und baute die Bauingenieurabteilung der USArmee, das Army Corps of Engineers, ein regionales Flutschutzsystem für New Orleans unter der Bezeichnung Lake Pontchartrain and Vicinity, Louisiana Hurricane Protection Project. Deiche und Flutschutzwände entlang des Seeufers im Norden, des Pumpenwerks und seiner Kanäle sowie der großen Schifffahrtskanäle wie dem Industrial Canal sollen Sicherheit vor zukünftigen extremen Klimaereignissen bieten (US House of Representatives 2006: 51). Dieses System versagte während des Orkans Katrina, in der Folge waren Teile der Stadt bis zu drei Wochen lang überschwemmt (vgl. Freudenburg/Gramling/Laska et al. 2009). Das stehende Hochwasser schädigte Bauten außen wie innen. Temporäre Pumpen hatten fast das gesamte Wasser aus der Stadt herausgepumpt, als Orkan Rita wenige Tage später zu intensiven Niederschlägen und erneutem Hochwasser führte.

Mehr als 105.000 Wohneinheiten allein in Orleans Parish wurden durch das Hochwasser schwer geschädigt - eine Zahl, die die Gesamtzahl der Schäden in den auch von Orkan Katrina betroffenen Staaten Mississippi, Alabama, Texas und Florida übertraf (Wagner/Frisch/Fields 2008: 40). Katrina bewirkte dadurch den größten Schadensfall dieser Art in der Geschichte der USA (GNOCDC 2008: 17). Diese massive Zerstörung und Beschädigung von Wohnraum wurde zum zentralen Thema des Wiederaufbaus und die hierbei beobachteten Schwierigkeiten lassen sich zum Teil mit der problembehafteten Krisenreaktion des Staates begründen (US House of Representatives 2006). Bundesstaatliche Wiederaufbauförderung wurde 2005 und 2006 in Höhe von über $\$ 100 \mathrm{Mrd}$. durch den Kongress bereitgestellt (Kromm/Sturgis 2007). New Orleans war jedoch bereits vor Katrina eine schrumpfende Stadt, zerrüttet durch ethnische Segregation und dem Wegzug weißer Bewohner in umliegende Vororte im Verlauf der White Flight. Allein im Lower Ninth Ward nahm die Bevölkerung von 33.000 in 1960 auf 19.500 vor Katrina ab (Campanella 2008: 153). Vor dem Orkan lebte beinahe ein Drittel der Gesamtbevölkerung der Stadt unterhalb der Armutsgrenze. 2010 erreichte die Bevölkerung wieder $80 \%$ - sie lebte jedoch ungleichmäBig (asymmetrisch) über die Stadt verteilt (GNOCDC 2000; 2003a; 2003b; 2010).

Das Lower Ninth Ward ist ein Teil von Orleans Parish und grenzt flussabwärts an das benachbarte St. Bernard Parish. Ursprünglich bildete das Ninth Ward einen zusammenhängenden Teil der Stadt, bevor es durch den Bau des Industrial Canal in Upper und Lower Ninth Ward getrennt wurde. Planung für eine Wohnnutzung in diesem Bereich fand bereits in der Mitte des 19. Jahrhunderts statt (Campanella 2008: 151). Das Lower Ninth Ward erstreckt sich über 2,3 Quadratmeilen und ist im Wesentlichen als Wohngebiet ausgewiesen, mit gewerblichen Nutzungen entlang der 
Hauptverkehrsadern St. Claude Avenue und Claiborne Avenue, die parallel zum Mississippi verlaufen. Es besteht aus zwei Neighborhoods, der Holy Cross Neighborhood und der Lower 9 Neighborhood, sowie einer Militäreinrichtung der Nationalgarde, Jackson Barracks. Die Holy Cross liegt auf dem natürlichen Deich, der sechs bis acht Fuß $(1,80-2,50 \mathrm{~m})$ oberhalb des Meeresspiegels entlang des Mississippi verläuft, und genießt als bauliches Ensemble Denkmalschutz. Hier befinden sich ab dem späten 19. Jahrhundert gebaute Wohnhäuser mit erhöhtem Erdgeschoss. Die Lower 9 wurde nach nach dem Bau des Industrial Canal trockengelegt. Dieser Bereich wurde als einer der letzten Teile im Stadtgebiet mit Häusern bebaut, mit Erdgeschossen auf Betonplatten auf Umgebungsniveau, das heißt zwei bis vier Fuß (60-120 cm) unterhalb des Meeresspiegels.

Im Lower Ninth Ward führte insbesondere der Zusammenbruch der Flutschutzmauern entlang des Industrial Canal, eines kommerziell genutzten Schifffahrtskanals innerhalb der Stadtgrenzen, zu massiven Überflutungen und Zerstörungen von Wohnbauten. Bewohnern wurde aufgrund der erneuten Überschwemmung durch Orkan Rita im Herbst 2005 erst spät gestattet, zurückzukehren. Gleichzeitig verblieben viele Bewohner nach der Katastrophe in Evakuierungszentren wie Houston. Laut FEMA waren $80 \%$ der Häuser beschädigt oder zerstört (Cuddeback/Bosworth 2008: 83 f.). Von insgesamt knapp 8.000 Wohneinheiten vor Katrina waren 3.671 von ihren Eignern bewohnt, 3.131 von Mietern. Vor Katrina lebten in der Holy Cross Neighborhood 2.240, in der Lower 9 Neighborhood 5.363 Haushalte. 2010, fünf Jahre nach Katrina, waren insgesamt kaum mehr als 30\% der Haushalte zurückgekehrt (Plyer 2010: 2). Jedoch zeigt die Forschung hier ein Ungleichgewicht auf: Im Juni 2010 betrug die Zahl der Haushalte in der Holy Cross 1.226 und in der Lower 9 sogar nur 1.271 (Plyer 2010: 2). Dies kann durch stadtweite Beobachtungen ergänzt werden: Die Bereiche der Stadt entlang der natürlichen Mississippi-Deiche waren weniger geschädigt und aktiver und konnten mit geringerem Aufwand wiederaufgebaut werden als die tiefer liegenden Bereiche (Colten 2005: xvii ff.). Die Ursachen dieser ungleichmäßigen Rückkehr sind jedoch nicht ausschließlich in der Lage begründet, wie die Fallstudie dieses Beitrags aufzeigen soll. Die beiden Karten zeigen die Dauer der Überflutung im Orleans Parish sowie die nach Katrina verbliebene Bebauung im Lower Ninth Ward.

\section{Methodologie}

In diesem Beitrag werden qualitative und quantitative Methoden $\mathrm{zu}$ einem integrierten Forschungsdesign verknüpft, um eine empirische Fallstudie im Lower Ninth Ward zu erstellen. Es wurden zunächst qualitative ethnographische Interviews geführt; sie wurden als Ausgangspunkt für eine quantitative fragebogenbasierte Untersuchung genutzt. Deren Ergebnisse wurden schließlich durch qualitative leitfadenbasierte Experteninterviews ergänzt. Diese abschließende Methode diente zur ,Vertiefung und Überprüfung von Ergebnissen“ (Miles/Huberman 1994: 41 f.). Durch diese Methodenmischung können breitere oder vollständigere Ergebnisse hinsichtlich des Forschungsgegenstandes erzielt werden. Dabei dient eine Fallstudie dazu, einen Einzelfall zu rekonstruieren, wobei als Fall „Personen, eine Familie,
Karte 1: Orleans Parish: Dauer der Überflutung (Quelle: Wallace Roberts \& Todd, LLC (2006: Fig. 4); nachbearbeitet)

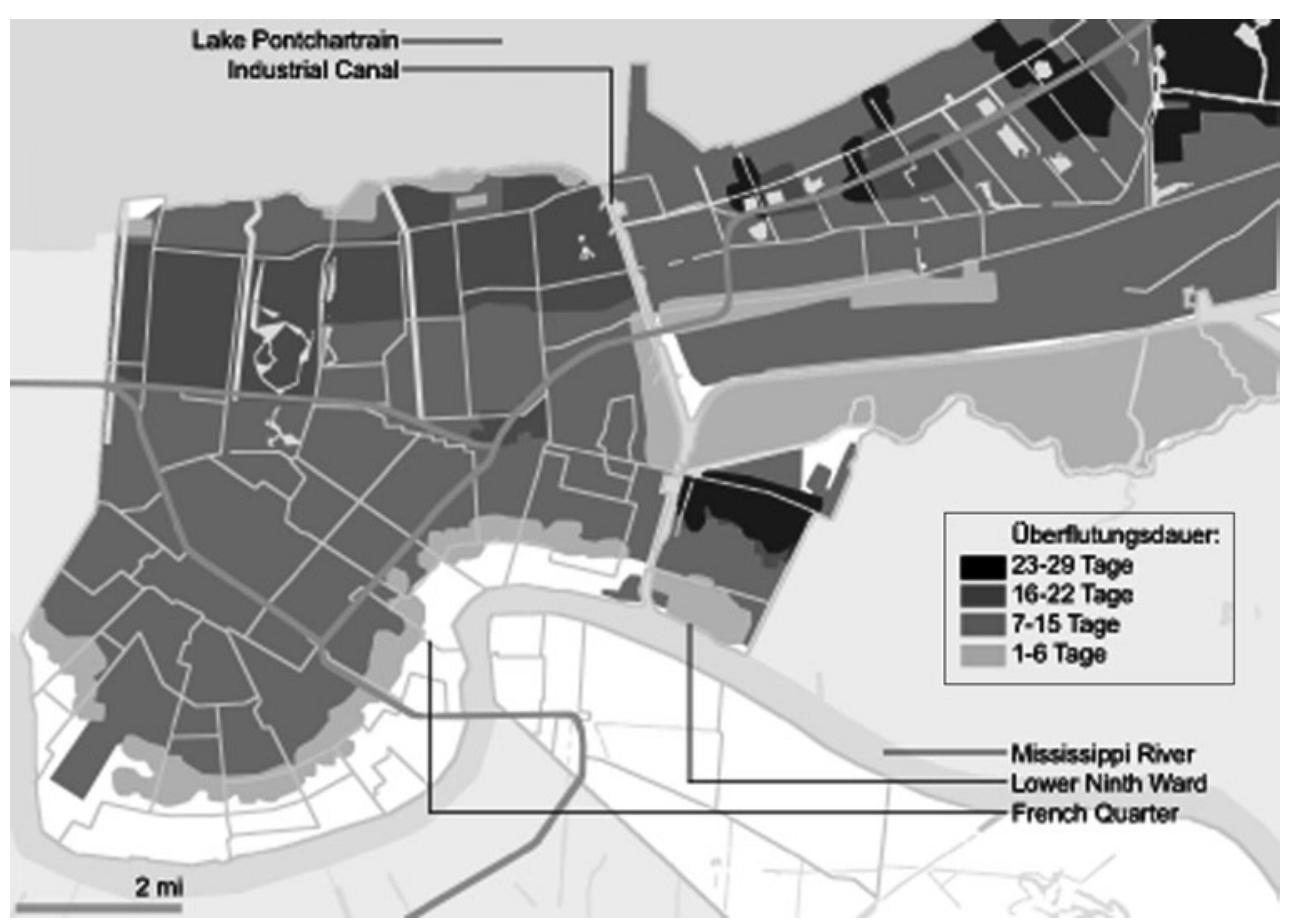




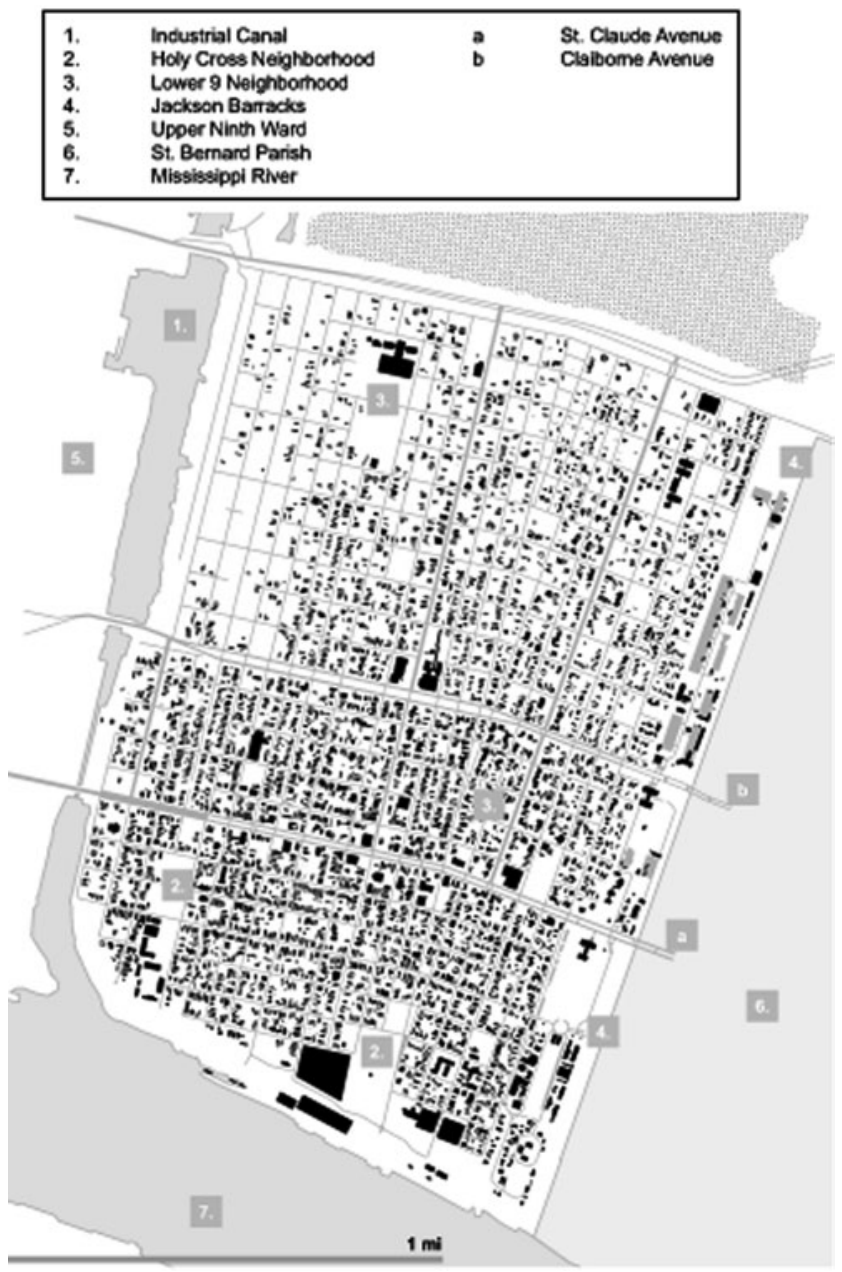

Karte 2: Verbliebene Bebauung im Lower Nith Ward nach Katrina (Quelle: Feireiss (2009: 37); H3 Studio Project Team (2007: 6.17); City of New Orleans (o. J.b: 16); Regional Planning Commission (2009); nachbearbeitet und überlagert)

eine Gemeinschaft oder eine Institution“ (Flick 2012: 177) verstanden werden können.

Insbesondere in der Katastrophenforschung werden gesellschaftliche Gruppen wie die Community als eine allgemeingültige Form sozialen Lebens und Handelns und damit als geeignete Forschungseinheit angesehen (Dynes 1998: 109). Räumlich betrachtet kann eine Community definierte Ausmaße oder territoriale Grenzen in Zusammenhang mit einem bestimmten Grad an permanenter Besiedlung aufweisen (Dynes 1998: 124). Das Konzept der Community kann im Fall der amerikanischen Stadt durch den Begriff Neighborhood ergänzt werden: die Neighborhood wurde erstmals im späten 19. Jahrhundert als grundlegende Einheit innerhalb der Stadt bezeichnet (Williams 1985: 29 f.). Insbesondere die funktionale Differenzierung des Stadtraumes wurde dahingehend interpretiert, dass die Stadt eine Fügung interagierender Communities darstelle, wobei Neighborhoods als Orte betrachtet werden, in denen Menschen sich zur Community zusammenfinden. Zudem sind es Charakteristiken wie Klasse, Ethnie, Geschlecht oder verschiedene Lebensweisen, die Neighborhoods voneinander unterscheiden können (Gottdiener/Hutchison 2006: 141).

Die ethnographischen Interviews wurden vom Autor in New Orleans und dem Lower Ninth Ward während des ersten Forschungsaufenthaltes in 2007 durchgeführt. Der Zweck bestand darin, genaueres über den Forschungsgegenstand (das Lower Ninth Ward als Quartier der USamerikanischen Stadt New Orleans, die Verwundbarkeit der Bewohner) zu erfahren. Drei Interviews mit Mitarbeitern von Non-Profit-Organisationen (Holy Cross Neighborhood Organization, ACORN, beide in New Orleans, und United Way in Houston, Texas) trugen wesentlich zur Durchführung der darauffolgenden quantitativen fragebogenbasierten Untersuchung bei. Jedoch musste die Situation vor Ort berücksichtigt werden. Zum einen bestand die Herausforderung darin, dass 2007 viele Betroffene noch nicht zurückgekehrt waren. Manche Betroffene befanden sich zwar wieder in New Orleans, aber außerhalb des Lower Ninth Ward, da ihr Wohnraum dort noch beschädigt oder zerstört war. Auch musste darauf Rücksicht genommen werden, dass Betroffene noch traumatisiert waren oder nach wie vor mit der Bewältigung ihrer Umstände zu kämpfen hatten. Zum anderen bestand ein Interesse daran zu erfahren, warum genau Betroffenen eine Rückkehr möglich oder nicht möglich war. 2007 war der Aufenthalt des Autors vor Ort auf zehn Wochen begrenzt. Aus diesen Gründen wurde, ausgehend von den Interviewten der ersten ethnographischen Interviewrunde, nach Möglichkeiten einer Umsetzung der quantitativen Befragung gesucht.

Daher wurde eine Teilerhebung der angestrebten Grundgesamtheit - in diesem Fall der Bewohner des Lower Ninth Ward-durchgeführt. Die Auswahl basierte nicht auf einem Zufallsprozess, sondern war eine bewusste Auswahl und wurde als Schneeballverfahren geplant, das heißt, es wurde ,ausgehend von einer Person die von dieser benannten Personen befragt" (Schnell/Hill/Esser 2011: 294). Die gesamte Auswahlgruppe umfasste 75 Personen, davon 12 in Houston. Die Fragebögen wurden in Anwesenheit des Autors ausgefüllt. Die fragebogenbasierte Untersuchung diente dazu, die Verwundbarkeit betroffener Bewohner des Lower Ninth Ward zu ermitteln und mit bestehenden Daten größerer Erhebungen zu vergleichen. Es wurden zurückgekehrte und nicht-zurückgekehrte (in Houston verbliebene) Betroffene vergleichend erfasst, um die Bereitschaft zur Rückkehr und damit zum Wiederaufbau zu untersuchen. Dies begründet die Inklusion in Houston verbliebener Langzeitevakuierter aus dem Lower Ninth Ward in die Fallstudie.

Die leitfadenbasierten Experteninterviews wurden 2009 in New Orleans und Washington, D.C. mit Personen geführt, die ,als Experte für ein bestimmtes Handlungsfeld [bzw.] als Repräsentant einer Gruppe“ (Flick 2012: 214) 
identifiziert wurden. Die Befragten waren „Mitarbeiter einer Organisation in einer spezifischen Funktion" (Flick 2012: 215). Die Experteninterviews wurden dafür genutzt, die vorangegangene quantitative Methode zu ergänzen. Die Interviewpartner wurden gezielt ausgewählt. Da der Forschungsaufenthalt 2009 wieder auf zehn Wochen begrenzt war, wurde die Möglichkeit der Zeitersparnis mittels Leitfadeninterview genutzt. Dabei stellten „mehr oder minder offen formulierte Fragen“ (Flick 2012: 221 f.) die Grundlage der Interviews dar. Die Fragen standen mit dem theoretischen Ansatz und der Fragestellung in Bezug. Es bot sich dabei die Möglichkeit, das Interview hinsichtlich des Themas zu steuern und nötigenfalls Schwerpunkte zu setzen (vgl. Flick 2012: 268 f.). Die Daten wurden als digitale Audiodateien aufgezeichnet, durch Transkription wortgetreu aufbereitet und in einen eigenständigen Text verwandelt.

Am Interviewmaterial wurde eine qualitative Inhaltsanalyse vorgenommen. Analytische Kategorien wurden aus dem theoretischen Ansatz heraus entwickelt und ,an das Material herangetragen“ (Flick 2012: 409) und nicht aus dem Material heraus abgeleitet. Die Ergebnisse der Analyse wurden ,,in Bezug auf die Fragestellung interpretiert“ (Flick 2012: 410). Eine Zusammenfassung wurde mittels Paraphrasen erstellt, wobei Reduktionsschritte Kürzungen und Bündelungen ermöglichten. Über eine explizierende Inhaltsanalyse wurde hierbei ergänzendes zusätzliches und kontextrelevantes Material hinzugefügt. Mittels einer weiteren Kontextanalyse wurden dabei „Informationen außerhalb des Textes“ (Flick 2012: 414) für explizierende Paraphrasen genutzt. Dies stellte sich als hilfreich heraus, da die Auswertung inhaltlich orientiert war und die Transkripte umfangreiche Texte darstellten (vgl. Flick 2012: 410 ff.). In den Experteninterviews wurden die institutionellen Planungen und Programme im Wiederaufbau von der bundesstaatlichen zur staatlichen und lokalen, stadtplanerischen Ebene nachgezeichnet. Besondere Aufmerksamkeit erhielten dabei Interaktionen mit Non-Profit-Organisationen, die beim Wiederaufbau in der Forschungseinheit tätig sind.

\section{Ergebnisse der empirischen Fallstudie}

\subsection{Quantitative fragebogenbasierte Untersuchung}

Die Daten der quantitativen Befragung wurden mit verfügbaren demographischen Daten (GNOCDC 2000; GNOCDC 2003a; GNOCDC 2003b) sowie einer weiteren, größeren Studie über Katrina-Evakuierte in Houston (Wilson/Stein 2006) verglichen. Die offiziellen, vor Katrina erhobenen Daten weisen darauf hin, dass seinerzeit die Bewohnerschaft des Lower Ninth Ward zu 95\% schwarz (African-American) war, $30 \%$ ein Einkommen von weniger als $\$ 15.000$ jährlich hatten, Frauen eine knappe Mehrheit ausmachten und mehr als $20 \%$ älter als 50 Jahre waren. Der Studie von Wilson und Stein zufolge waren $98 \%$ der Evakuierten in Houston schwarz, ein wesentlicher Anteil hatte ein Einkommen von weniger als \$15.000 jährlich und viele würden wahrscheinlich nicht nach New Orleans zurückkehren, da nur wenige von ihnen Hauseigentümer gewesen sind (Wilson/Stein 2006: 7). Die in der empirischen Studie des Autors Befragten umfassten $77 \%$ schwarze Bewohner, $16 \%$ mit einem Einkommen von weniger als 15.000 jährlich. $72 \%$ waren weiblich, $60 \%$ waren älter als 50 Jahre.

Bestandteil der quantitativen Befragung waren Faktoren, die die Rückkehr beeinflussen, wie beispielsweise die Wohnform und orkanbedingte Schäden. Vor Katrina lebten 71 Befragte in einem eigenen Haus, nach dem Orkan waren es nur noch 19. Mietwohnungen oder Wohnwägen zählten zu den möglichen Alternativen. 60 Teilnehmer waren vor Katrina Hauseigentümer, 11 waren Mieter. Nach der Katastrophe nahm die Zahl der Hauseigentümer auf $41 \mathrm{ab}$ und die der Mieter auf 19 zu. 42 Befragte gaben an, ihr Zuhause sei „stark beschädigt“, 28 meldeten „komplett zerstört“. 14 Personen waren nach Hause zurückgekehrt, 47 hatten noch nicht wieder daheim einziehen können. Als Gründe, die eine Rückkehr erschwerten, wurden „kein Geld“ sowie „schlechte Erinnerungen“, „Abwesenheit von Familie und Freunden“ und „mangelnde Sicherheit" genannt. Die Befragten gaben außerdem an, dass es ,sehr schwierig“ gewesen sei, institutionelle Hilfen zu bekommen, und zwar aufgrund der Antragstellung an Versicherungen, die bundesstaatliche Katastrophenschutzbehörde FEMA (Federal Emergency Management Agency) oder für Baugenehmigungen.

Die zurückgekehrten Bewohner waren mehrheitlich Hauseigentümer, die zum Zeitpunkt der Befragung in Apartments oder anderen Alternativen wohnten. Unter den in Houston befragten waren mehrheitlich Mieter. Die Rolle der Verwundbarkeiten in Bezug auf Ethnie und Klasse sind hinreichend bekannt (vgl. IRDR 2011: 12). Wie die Wohnumstände der Betroffenen andeuten, hat diese sogar zugenommen. Bemerkenswert ist, dass sowohl Mieter als auch Eigentümer Bedarf an alternativem Wohnraum hatten. Dies lag daran, dass Häuser und Wohnungen beschädigt oder zerstört oder während des Wiederaufbaus nicht bewohnbar waren. Zudem bestand das Problem des Zugangs zu institutionellen Hilfen. Diese Problemfelder bildeten den Hintergrund der Fragen in den nachfolgenden qualitativen Experteninterviews in Zusammenhang mit den Plänen und Programmen des Wiederaufbaus.

\subsection{Experteninterviews}

Mit Experteninterviews wurden (leitende) Mitarbeiter bundesstaatlicher und lokaler Institutionen sowie Non-ProfitOrganisationen, die sich beim Wiederaufbau im Lower 
Ninth Ward betätigen, befragt. Die hier verwendeten Interviews wurden mit einem Stabsmitarbeiter des Department of Homeland Security in Washington, D.C., dem Direktor für Immobilienstrategie von NORA in New Orleans, der leitenden Direktorin des Preservation Resource Center (PRC) sowie dem leitenden Direktor von lowernine.org geführt wobei es sich bei den letzten beiden um Non-Profit-Organisationen in New Orleans handelt, die Betroffenen bei ihren Wiederaufbaubemühungen halfen.

Wie das Interview mit einem Stabsmitarbeiter des Department of Homeland Security, dem für Katastrophenschutz zuständigen Bundesministerium in Washington, D.C., zeigt, gab es Gründe, den Staat Louisiana als Verwalter der Fördergelder für den Wiederaufbau von Wohnraum zu bestimmen. Das Instrument der Community Development Block Grants (CDBG) wurde im Fall von Orkan Katrina gesondert autorisiert und unterscheide sich dabei von einer typischen Verwendung dieses Instruments. Ziel sei es gewesen, dem Staat Louisiana Flexibilität bei der Verwendung der Gelder einzuräumen, da die Erkenntnis bestand, dass die Institutionen vor Ort eine bessere Vorstellung davon hätten, wie mit solchen Geldern umzugehen sei. Diese Entscheidung basiert auch auf dem Aktionsplan des Staates Louisiana, der als Grundlage der Fördergelder und nicht zuletzt des Road Home Program diente.

Der Direktor für Immobilienstrategie von NORA sieht diese Konstellation kritisch. Die Tatsache, dass Entscheidungen über das Road Home Program beim Staat Louisiana liegen, nennt er im Interview problematisch und unfair der Stadt New Orleans gegenüber. Die Gelder wurden zunächst an das Office of Community Development (OCD) weitergeleitet, das im Road Home Program damals eine lenkende Funktion einnahm. Die Kernaufgabe des Office of Community Development läge jedoch in der Entwicklung der ländlichen Bereiche Louisianas, das heißt nicht auf dem Gebiet der Stadtplanung. Die Möglichkeit der Entscheidung über die Verwendung der Gelder sollte aber bei denjenigen Körperschaften liegen, die letzten Endes die Nutznießer seien. Die Tatsache, dass die Gelder, um in die Stadt zu gelangen, durch den Staat fließen müssten, betrachtet der Interviewte als absurd. Daraus würden sich Folgeprobleme ergeben. ${ }^{5}$

Es wurde thematisiert, dass insbesondere Verzögerungen in der Antragstellung - der Fokus lag auf Hauseigentümern sowie die erst nach Anlaufen des Road Home Program ein-

\footnotetext{
${ }^{5}$ Ebenfalls in der Form eines Experteninterviews ausgeführt, jedoch nicht mit einem Mitglied einer staatlichen Institution oder einer Non-Profit-Organisation, liefert das Gespräch des Autors mit einem Journalisten der lokalen Tageszeitung in New Orleans folgende, alternative Erklärung: Zwischen der Gouverneurin des Staates Louisiana, Kathleen Blanco, und dem Bürgermeister der Stadt New Orleans, Ray Nagin, obwohl beide Mitglieder der Demokratischen Partei, herrschte ein gespanntes Verhältnis. Daher sei die Wahrnehmung, man könne den Wiederaufbau ohne Einflussnahme der Stadt vornehmen, möglicherweise als opportun betrachtet worden.
}

geführte Option des Grundstücksrückkaufs (Buyout) - dem Wiederaufbau in der Stadt entgegenstehen. Es sei wichtig gewesen, dass betroffene Bürger Geld für Reparaturen und Baumaßnahmen schnell erhielten. Jedoch sei insbesondere durch die komplexe Antragstellung des Road Home Program und die damit verbundenen Verzögerungen eine schnelle Förderung verhindert worden. Eine Rückkehr sei aber umso unwahrscheinlicher, wenn Betroffene mehrere Jahre lang auf Fördergelder warten müssten und dabei bereits an einem anderen Ort lebten. Von äußerster Bedeutung sei das Missverhältnis zwischen der Förderung für Hauseigentümer und dem für Mietraum gedachten Teil des Road Home Program, da in der Stadt New Orleans, anders als im Durchschnitt des Staates Louisiana, mehr Mieter als Hauseigentümer gelebt hatten.

Ein weiteres Problem war die Berechnung von Fördergeldern, die auf dem jeweiligen Grundstückswert vor Katrina basieren. Es wurde festgestellt, dass diese Methode für den Wiederaufbau nicht sinnvoll war: Da die zugeteilte Förderung die eigentlichen Kosten der Wiederinstandsetzung gerade bei niedrigen Grundstückswerten nicht decken konnte, hätten sich Betroffene eher für die Option auf den Rückkauf ihrer Grundstücke durch den Staat Louisiana entschieden, anstatt ihre Häuser wieder aufzubauen. Als Folge stünden nicht nur in ärmeren Vierteln der Stadt Grundstücke und Häuser leer. Sie seien auch, bedingt durch viele individuelle Entscheidungen zum Wiederaufbau oder Nicht-Wiederaufbau, beliebig in der Stadt verteilt. Dadurch ergeben sich unregelmäßige und ungeplante Muster des Leerstands, wie fehlende Zähne in einem Gebiss. Das Ziel von NORA ist es daher, diesen Leerstand planerisch zu kontrollieren. Hierzu diente das Lot Next Door Program. Hat im Rahmen dieses Programms ein betroffener Grundstücksbesitzer erfolgreich eine Wiederaufbauförderung beantragt und nutzt (aus welchen Gründen auch immer) die Rückkaufoption, kann NORA in der Folge über das Grundstück verfügen und es verkaufen. Dabei wird der Besitzer des direkt angrenzenden Nachbargrundstücks als Käufer präferiert. Dieser kann nun das leerstehende Grundstück seinem eigenen zuschlagen und es damit vergrößern. Der Leerstand im betroffenen Stadtteil wird dadurch rechnerisch vermindert. Im Idealfall kann eine Nachbarschaft mit 50\% Leerstand auf diese Weise ,stabilisiert' werden. Je mehr verbleibende Grundstücksbesitzer die von NORA verwalteten leerstehenden Grundstücke kaufen, desto mehr geht der Leerstand rechnerisch zurück und vakante Gebäude werden aus dem Stadtbild entfernt. Dadurch wird ein Rückgang an baulicher Dichte in Folge der Katastrophe nicht verhindert, jedoch planerisch kontrolliert oder zumindest begleitet.

Aber es gibt einen Haken: Kritisch ist ein Leerstand von über $50 \%$, bei dem die Wirksamkeit des Lot Next Door Program seitens NORA in den betreffenden Nachbarschaften als begrenzt angesehen wird. Es gibt in diesen Fällen keine 
genügend große Zahl an zurückgekehrten Bewohnern, die das Programm so nutzen könnten, dass der unregelmäßige Leerstand effektiv bekämpft werden könne. Dies betrifft Neighborhoods mit massiven Orkanschäden, Bevölkerungsverlusten durch Langzeitevakuierung sowie wirtschaftlichen Problemen. Auch der Citywide Recovery Plan stellt fest, dass in solchen Teilen der Stadt nicht flächendeckend, sondern punktuell agiert werden sollte, da sowohl Ressourcen als auch Initiativen begrenzt seien. Gerade deshalb sei es auch wichtig, auf den Kontext zu reagieren und bereits vor Katrina einsetzende Schrumpfungsprozesse zu berücksichtigen sowie der Situation angemessene Lösungen vorzuschlagen, beispielsweise auf dem bestehenden Parzellensystem maßstabsgerecht Wiederaufbau zu betreiben (Infill-Development).

Trotz dieser Schwierigkeiten, die von institutionellen Initiativen des Wiederaufbaus zum Teil mitverursacht wurden, ist der Einsatz von Non-Profit-Organisationen im Wiederaufbau von New Orleans nach Katrina hervorzuheben. Gerade in benachteiligten Vierteln zeigen sie eine außerordentliche Hilfsbereitschaft. Zwei weitere Interviews sollen verdeutlichen, wie die Interaktionen zwischen den staatlichen Institutionen und diesen Organisationen den Wiederaufbau beeinflussen. Die leitende Direktorin des Preservation Resource Center (PRC) beschreibt deren Wiederaufbauaktivität im Fallstudienbereich. Man habe sich bereits vor Katrina insbesondere mit den historischen Bauten der Holy Cross Neighborhood beschäftigt. Mittlerweile kauften sie auch Grundstücke von NORA, jedoch abhängig von eigenen Ressourcen. Das Lot Next Door Program sei nicht nur geeignet, Hauseigentümern zu helfen, ihre Grundstücke zu vergrößern und deren Wert zu steigern, dadurch erhöhe sich auch die Attraktivität des jeweiligen Viertels. In den am schwersten betroffenen Vierteln werde die Notwendigkeit der Bündelung von Maßnahmen akzeptiert, diese dürften jedoch nicht unveränderbar sein, sondern müssten flexibel auf entsprechende Entwicklungen reagieren können.

Der leitende Direktor von lowernine.org, einer NonProfit-Organisation im Lower Ninth Ward, beschreibt die Eigenschaften der Betroffenen, die von ihnen Unterstützung im Wiederaufbau ihrer Häuser erhalten. Diese wollten zurückkehren, verfügten aber nicht über ausreichende Fördergelder durch das Road Home Program, auch wegen der niedrigen Grundstückswerte. Dieser Umstand werde zum Beispiel durch fehlende Versicherungen gegen Wind oder Flut noch verstärkt. Zu den Klienten gehörten Anwohner mit niedrigen Einkommen, Senioren, Menschen mit Behinderungen sowie Frauen mit Kindern. $\mathrm{Zu}$ den wiederhergestellten Gebäuden zählten Einfamilienhäuser wie auch Zweifamilienhäuser, bei denen eine Wohneinheit vom Eigentümer bewohnt würde und die andere von einem Mieter - als Small Rental Property eine typische Wohnsituation in New Orleans.
Hier lässt sich die schwache Verbindung oder sogar die Entkopplung zwischen institutionellen Plänen und Programmen des Wiederaufbaus und der Verwundbarkeit Betroffener erkennen. Die auf Armut und Ethnie basierende Verwundbarkeit der Bewohner des Lower Ninth Ward ist hierbei von wesentlicher Bedeutung. Durch sie hat das Road Home Program nur begrenzte Wirksamkeit. Die durch niedrige Grundstückswerte und Versicherungsprobleme verschärften Finanzierungslücken stellen wesentliche Hindernisse im individuellen Wiederaufbau dar, die auch die Verfügbarkeit von Mietwohnraum betreffen. Non-Profit-Organisationen helfen den Bewohnern des Lower Ninth Ward zwar bei ihren Wiederaufbaumaßnahmen und interagieren mit den lokalen Institutionen. Bei niedriger Wiederbevölkerungsrate besitzen institutionelle Maßnahmen dennoch nur eine begrenzte Wirksamkeit. Denn für einen Planning District mit einer durchschnittlichen Rückkehrrate unterhalb von $50 \%$ sieht der Citywide Plan nur beschränkt Maßnahmen zum Wiederaufbau vor. Auch innerhalb des Planning District ergibt sich ein gespaltenes Bild: Der bescheidene Erfolg der Holy Cross Neighborhood steht der Situation in der Lower 9 gegenüber, mit ihrem in weiten Teilen parkähnlichen, unbesiedelten Charakter mit vereinzelten, wenn auch prominenten Wiederaufbauinitiativen. Beide sind auf Wohl und Wehe miteinander verbunden.

\section{Schlussfolgerungen}

Wie die oben geschilderten Ergebnisse zeigen, reagierten die Pläne und Programme des Wiederaufbaus in New Orleans nach Orkan Katrina im Lower Ninth Ward nur sehr bedingt auf die städtebaulichen und sozialen Rahmenbedingungen und damit auf die Verwundbarkeit der Bewohner. Das Road Home Program mit seiner verzögerten und mangelhaften Wiederaufbauförderung, der Citywide Plan und das Lot Next Door Program sind nur begrenzt und unterschiedlich wirksam. Dies beeinflusste die Bereitschaft zur Rückkehr und zum Wiederaufbau wesentlich. Dabei können die tief liegenden Ursachen der Verwundbarkeit als Teil des Alltagslebens in der Stadt aufgefasst werden. Der höhere Anteil von Mietern im Vergleich zum landesweiten Durchschnitt in den Städten der USA ist ein Beispiel hierfür, ebenso die historische ethnische Ungleichheit. Zudem spielen Ort, Lage und Bauweise, zumindest im Fall eines Hochwassers, eine Rolle. Die historische und höher gelegene Holy Cross Neighborhood konnte besser Wiederaufbau betreiben als die moderne Lower 9 Neighborhood, die sich im Wesentlichen nur auf das Flutschutzsystem verlassen konnte, das bekanntlich versagte. Die Kombination dieser Aspekte trug zum asymmetrischen Wiederaufbau im Lower Ninth Ward bei. 
Während das Lot Next Door Program in der Holy Cross anscheinend hilfreich ist, führen die Grundstücksverkäufe (Buyouts) insbesondere im Lower Ninth Ward zu einem unregelmäßigen Muster des Leerstands.

Das Ergebnis im Lower Ninth Ward steht dem normativen Paradigma des Stafford Act, der geforderten Rückkehr zur Normalität, entgegen. Für verwundbare Bevölkerungsgruppen ist dieses Paradigma ohnehin schwer erreichbar, wenn überhaupt wünschenswert, gerade in Stadtteilen, die vor der Katastrophe von Abschwung und sozialen Problemen betroffen waren. Die Orientierung am Status quo ante beim Wiederaufbau trägt offenbar weder dazu bei, die Vulnerabilität betroffener Populationen zu vermindern, noch das wünschenswerte Leitbild des „Building Back Better“ umzusetzen. Gerade durch die Tatsache, dass Verwundbarkeiten eben ein Bestandteil des Alltagslebens sind, wird die Normalität selbst zu einem Zustand, der einer kritischen Bestandsaufnahme bedarf. Hier stellt sich die geforderte schnelle Rückkehr zu einem Zustand der Normalität als Ziel des Stafford Act als trügerisch heraus. Eine Rückkehr zur Normalität in dieser Form kann gar nicht zu einer Verminderung der Vulnerabilität beitragen.

Als Folge bleibt die Herausforderung, die Planung für den Wiederaufbau in und damit von Städten nach Katastrophen hinsichtlich der Verwundbarkeiten der Betroffenen zu optimieren. Die damit verbundenen Pläne und Programme müssen stärker auf diesen Kontext eingehen, als es in New Orleans der Fall war. Der Schwerpunkt auf Hauseigentümer müsste um eine besondere Förderung von Mietraum ergänzt werden (Small Rental Property). Dabei sollten bestehende Strukturen oder Schrumpfungsprozesse in Betracht gezogen werden. Dies betrifft auch legitime Forderungen nach Rückkaufoptionen, die jedoch koordiniert werden müssten, um die Nachhaltigkeit der Besiedlung des Stadtraums nicht in Frage zu stellen. Es ist wichtig, dass die Notwendigkeit der Änderung, Anpassung oder Verbesserung nicht bedeuten darf, dass Betroffene zu lange auf Förderung warten müssen. Beispielhaft kann hier die Interaktion von städtischen Institutionen und Non-Profit-Organisationen sein, wie das Beispiel New Orleans auch demonstriert. Da die Folgen des Hochwassers in sehr spezifischer Relation zu stadträumlichen Eigenschaften stehen, muss eine Übertragbarkeit der Ergebnisse auf andere Fälle (z. B. Erdbeben) geprüft werden. Eine Betrachtung des asymmetrischen Wiederaufbaus bietet sich aber nicht nur für die Ebene der Neighborhood oder des Quartiers an, sie ist auch stadtweit denkbar. Die Ergebnisse können auch als Grundlage für eine wissensbasierte Wiederaufbauplanung dienen, die sowohl gerecht als auch nachhaltig zur Minderung von Asymmetrien und Verwundbarkeit beim Wiederaufbau nach Katastrophen in Städten beiträgt.
Danksagung Der Dank des Autors gilt dem Deutschen Akademischen Austauschdienst, der die Feldforschung 2009 ermöglichte, sowie den anonymen Gutachtern für ihre hilfreichen Kommentare.

\section{Literatur}

Birkmann, J. (2006): Measuring vulnerability to promote disasterresilient societies: Conceptual frameworks and definitions. In: Birkmann, J. (Hrsg.): Measuring Vulnerability to Natural Hazards. Towards Disaster Resilient Societies. Tokyo, New York, Paris, 9-54.

Bohle, H.-G. (2001): Vulnerability and Criticality: Perspectives from Social Geography. In: Newsletter of the International Human Dimensions Programme on Global Environmental Change 2 (1), 3-5.

Campanella, R. (2008): Bienville's Dilemma. A Historical Geography of New Orleans. Lafayette.

Chambers, R. (1989): Editorial Introduction: Vulnerability, Coping and Policy. In: IDS Bulletin 20 (2), 1-7.

City of New Orleans (o. J.a): The Unified New Orleans Plan (UNOP). Citywide Strategic Recovery and Rebuilding Plan. New Orleans. http://quake.abag.ca.gov/wp-content/documents/resilience/ New\%20Orleans-FINAL-PLAN-April-2007.pdf (02.06.2014).

City of New Orleans (o. J.b): A Foundation for Recovery: Strategies for Neighborhood Stabilization. http://www.nolacitycouncil.com/ docs/resources/AFoundationForRecovery.ppt (02.06.2014).

City of New Orleans (2010): Plan for the $21^{\text {st }}$ Century. New Orleans 2030. New Orleans. http://www.nola.gov/city-planning/masterplan/ (02.06.2014).

Colten, C. (2005): An Unnatural Metropolis. Baton Rouge.

Cuddeback, M.; Bosworth, F. (2008): Rebuilding Community Block by Block. In: Cityscape 10 (3), 77-99.

Czerwinski S. (2007): Gulf Coast Rebuilding. Preliminary Observations on Progress to Date and Challenges for the Future. Testimony Before the Subcommittee on Disaster. Recovery, Committee on Homeland Security and Governmental Affairs Committee, U.S. Senate. http://gao.justia.com/department-of-housing-andurban-development/2007/4/gulf-coast-rebuilding-gao-07-574t/ (12.08.2014)

Dombrowsky, W. (1998): Again and again: Is a disaster what we call a „disaster"? In: Quarantelli, E. (Hrsg.): What is a disaster? Perspectives on the question. New York, 19-30.

Dynes, R. (1998): Coming to terms with community disaster. In: Quarantelli, E. (Hrsg.): What is a disaster? Perspectives on the question. New York, 109-126.

Dynes, R. (2006): Social Capital: Dealing with Community Emergencies. In: Homeland Security Affairs 2 (2), 5.

Feireiss, K. (2009): Architecture in Times of Need. Make It Right. Rebuilding New Orleans' Lower Ninth Ward. New York.

Flick, U. (2012): Qualitative Sozialforschung. Eine Einführung. Reinbek.

Freudenburg, W.; Gramling, R.; Laska, S.; Erikson, K. (2009): Catastrophe In The Making. The Engineering of Katrina and the Disasters of Tomorrow. Washington, DC.

Gaillard, J. C.; Glantz, M.; Kelman, I.; Wisner, B.; Delica-Willison, Z.; Keim, M. (2014): Taking the ,naturalness' out of natural disaster (again). In: Natural Hazards Observer 38 (3), 1-14.

GNOCDC - Greater New Orleans Community Data Center (2000): Percent African American by Census block group in Orleans Parish. http://gnocdc.org/maps/race/pdfs/african_american.pdf (02.06.2014) 
GNOCDC - Greater New Orleans Community Data Center (2003a): Income \& poverty comparison for neighborhoods within Orleans Parish. http://www.gnocdc.org/xls/nbhd_income.xls (02.06.2014).

GNOCDC - Greater New Orleans Community Data Center (2003b): People \& household characteristics comparison for neighborhoods within Orleans Parish. http://www.gnocdc.org/xls/nbhd_people. xls (02.06.2014).

GNOCDC - Greater New Orleans Community Data Center (2008): The New Orleans Index. Anniversary Edition. Three Years After Katrina. New Orleans.

GNOCDC - Greater New Orleans Community Data Center (2009): The New Orleans Index. New Orleans.

GNOCDC - Greater New Orleans Community Data Center (2010): The New Orleans Index at Five: Reviewing Key Reforms After Hurricane Katrina. New Orleans.

Gottdiener, M.; Hutchison, R. (2006): The New Urban Sociology. Boulder.

H3 Studio Project Team (2007): A Framework for Sustainable Resilience in the Lower Ninth Ward. http://willdoo-storage.com/Plans/ D8/District 08 Lower9th Chapter 01 Introduction to the District.pdf (03.06.2014).

Hassenpflug, D. (2006): Reflexive Urbanistik. Reden und Aufsätze zur europäischen Stadt. Weimar.

Hassenpflug, D. (2009): Der urbane Code Chinas. Basel.

IRDR - Integrated Research of Disaster Risk (2011): Forensic investigations of disasters: The FORIN Project. Beijing.

Kromm, C.; Sturgis, S. (2007): Blueprint for Gulf Renewal. Durham, NC.

Louisiana Recovery Authority (2006): Migration Patterns - Estimates of Parish Level Migrations due to Hurricanes Katrina and Rita. http://popest.org/popestla2006/files/MigrationReport_FINv4.pdf (03.06.2014)

Miles, M.; Huberman, A. (1994): Qualitative Data Analysis. An Expanded Sourcebook. Thousand Oaks.

Pelling, M. (2003): The Vulnerability of Cities. Natural Disasters and Social Resilience. London.

Perry, R.; Quarantelli, E. (Hrsg.) (2005): What Is a Disaster? New Answers to Old Questions. Philadelphia.

Plyer, A. (2010): Neighborhood Recovery Rates. https:/gnocdc. s3.amazonaws.com/reports/GNOCDC_NeighborhoodRecoveryJune2010.pdf (12.08.2014)
Pohl, J. (2010): Wiederaufbau nach dem Erdbeben - Perspektiven für Haiti. In: Aus Politik und Zeitgeschichte 28-29, 10-17.

Putnam, R. D. (2007): Bowling Alone: America's Declining Social Capital. In: Le Gates, R.; Stout, F. (Hrsg.): The City Reader. New York, 121-129.

Regional Planning Commission (2009): New Orleans Council District C: LLT Properties, April 17th 2009. http://bucrp.uno.edu/docs/ CouncilDistrictC24X36.pdf (12.08.2014).

Rodriguez, H.; Quarantelli, E.; Dynes, R. (2007): Handbook of Disaster Research. New York.

Schnell, R.; Hill, P.; Esser, E. (2011): Methoden der empirischen Sozialforschung. München.

Stallings, R. (1998): Disaster and the theory of social order. In: Quarantelli, E. (Hrsg.): What is a disaster? Perspectives on the question. New York, 127-145.

Stallings, R. (2005): Disaster, crisis, collective stress and mass deprivation. In: Perry, R.; Quarantelli, E. (Hrsg.): What is a disaster? New answers to old questions. Philadelphia, 237-274.

US Census (2012): State \& County Quick Facts. http://quickfacts.census.gov (04.05.2014).

US House of Representatives (2006): A Failure of Initiative: Final Report of the Select Bipartisan Committee to Investigate the Preparation for and Response to Hurricane Katrina. Washington, DC.

Vale, L.; Campanella, T. (2005): The resilient city: How modern cities recover from disaster. Oxford.

Wagner, J.; Frisch, M.; Fields, B. (2008): Building Local Capacity: Planning for Local Culture and Neighborhood Recovery in New Orleans. In: Cityscape 10 (3), 39-55.

Wallace Roberts \& Todd, LLC (2006): Action Plan for New Orleans: The New American City. http://www.npr.org/documents/2006/jan/ CityPlanningFinalReport.pdf (05.06.2014).

Weiss, N. (2007): Rebuilding Housing After Hurricane Katrina: Lessons Learned and Unresolved Issues. http://www.hlswatch.com/ sitedocs/RL33761.pdf (02.06.2014).

Williams, M. (1985): Neighborhood Organizations. Seeds of a New Urban Life. Westport.

Wilson, R.; Stein, R. (2006): Katrina evacuees in Houston: One-year out (white paper). http://brl.rice.edu/Katrina/White_Papers/ White Paper 98 06.pdf (02.06.2014). 